\title{
Muso franco e maronè: dubbi lessicali da un Figaro all'altro
}

\author{
Paolo D'Achille
}

PUBBLICATO: 12 NOVEMBRE 2019

\section{Quesito:}

Ci sono pervenute due richieste di chiarimento sul significato dell'espressione muso franco, che si trova nel libretto delle Nozze di Figaro, e del termine maroné, documentato nel Barbiere di Siviglia.

\section{Muso franco e maroné: dubbi lessicali da un Figaro all'altro}

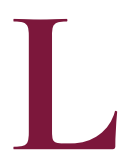

e domande vengono l'una da São Paulo, Brasile, e l'altra da Berlino e a proporcele sono due persone impegnate nella traduzione dei due libretti. Prima di rispondere, ritengo opportuna qualche considerazione di carattere generale.

Poiché ormai un po' dappertutto le opere liriche vengono eseguite nella lingua originale, la traduzione dei libretti non è più quella ritmica che sostituiva sulla scena le parole del testo musicato, al quale necessariamente non era troppo fedele (ciò vale sia per le traduzioni dei libretti in italiano, sia per le versioni in italiano di opere francesi o tedesche), ma viene predisposta per essere letta, o sui programmi di sala, o sui testi che corredano i dischi (l'uso, in questo caso, risale già all'epoca del vinile), o nei sottotitoli di riprese filmate in $\mathrm{dvd}$ o in trasmissioni televisive, o, infine, nei sopratitoli che a teatro scorrono sopra il palcoscenico (o altrove). Da questo deriva la tendenza a una maggiore aderenza al testo, che spiega la necessità dei traduttori di comprendere appieno il significato di tutte le parole.

Il carattere particolare sia della lingua dell'opera lirica (melodramma serio o opera buffa che sia) sia dell'esecuzione vocale (non tutti gli interpreti lirici fanno capire bene le parole che pronunciano cantando) rende i testi dei libretti italiani non del tutto comprensibili neppure agli italiani, e oggi meno di ieri, tanto che ormai i sottotitoli in italiano si usano anche per le opere italiane trasmesse in Italia. Se è vero che il senso generale del testo è per noi italiani abbastanza chiaro, è altrettanto vero che spesso ci accontentiamo di questa comprensione di massima, senza porre attenzione proprio a tutte le parole, alcune delle quali restano incomprensibili, tanto più alle giovani generazioni, a cui la scuola non garantisce più la dimestichezza con la lingua della tradizione poetica a cui quella dell'opera lirica è indissolubilmente legata.

Nei casi in questione, però, il discorso è parzialmente diverso. Siamo alle prese con i libretti di due opere che rientrano nel genere comico (commedia musicale o opera buffa che sia), tratti entrambi da due commedie francesi di Beaumarchais, Le nozze di Figaro di Mozart (libretto di Lorenzo Da Ponte, prima esecuzione a Vienna nel i786) e Il barbiere di Siviglia di Rossini (libretto di Cesare Sterbini, prima esecuzione a Roma nel ı8ı6, col titolo Almaviva, ossia L'inutil precauzione) e i testi del genere comico sono più aperti all'uso contemporaneo, e quindi disponibili ad accogliere dialettismi e forestierismi, come sono appunto l'espressione e la parola di cui ci stiamo occupando.

L'espressione muso franco compare nell'aria di Figaro che chiude il primo atto (la celebre Non piu andrai, farfallone amoroso), in cui il protagonista si rivolge al paggio Cherubino prospettandogli le difficoltà della vita militare a cui sembra costretto: 
Tra guerrieri, poffarbacco! / Gran mustacchi, stretto sacco, / schioppo in spalla, sciabla al fianco, / collo dritto, muso franco, / un gran casco, o un gran turbante, / molto onor, poco contante! // Ed invece del fandango, / una marcia per il fango. / Per montagne, per valloni, / con le nevi e i sollioni. / Al concerto di tromboni, / di bombarde, di cannoni, / che le palle in tutti i tuoni / all'orecchio fan fischiar.

Anche marroné ricorre in bocca a Figaro, in un recitativo della scena 3 dell'atto II, quando il barbiere convince Don Bartolo a farsi la barba quel giorno con la scusa che il successivo ha una serie di impegni:

figaro: Oggi non vuol?... dimani / non potrò io.

bartolo:

Perché?

figaro:

Perché ho da fare / a tutti gli ufficiali / del nuovo reggimento barba e testa... / Alla marchesa Andronica / il biondo perucchin coi maronè... / Al contino Bombè / il ciuffo a campanile... / Purgante all'avvocato Bernardone / che ieri s'ammalò d'indigestione...

Ora, per quanto riguarda muso franco il GDLI (oggi consultabile sul sito dell'Accademia) non registra l'espressione né s.v. muso né s.v. franco, ma riporta (s.vv. intimare e pedemonte) un esempio di Ippolito Nievo (posteriore quindi a quello dapontiano):

La gente del paese, benché non fosse rissosa e manesca al pari di quella del pedemonte, aveva muso franco abbastanza per imbeversi spesse volte delle sentenze di cancelleria, e per dar la berta al cavallante che le intimava.

Considerando l'origine veneta sia di Da Ponte sia di Nievo, viene subito da pensare che si tratti dell'inserimento in un contesto italiano di un'espressione dialettale. In effetti è così: muso franco è attestato (come risulta da Google Libri) in commedie in dialetto veneziano di Pietro Chiari (I76o) e di Alessandro Pepoli (I788). Anche il Dizionario del dialetto veneziano di Giuseppe Boerio (Venezia, Santini, I829, p. 370) registra muso franco, nel senso di "Viso asciutto o aperto, cioè Che mostra franchezza". Del resto, il GRADIT registra tra le varie accezioni di franco anche quella di 'sicuro di sé, disinvolto; risoluto, deciso' e di muso non dà solo il solo il significato di 'parte anteriore prominente della testa di animale che include la bocca', ma anche quello, in senso spregiativo o scherzoso, di 'faccia, volto di persona'; significato, questo, ben diffuso nei dialetti, in particolare nel veneziano, come risulta dal Vocabolario del veneziano di Carlo Goldoni di Gianfranco Folena (Roma, Istituto della Enciclopedia Italiana, I993, pp. 382-383).

A proposito di Goldoni, è proprio il grande commediografo che offre, nel libretto di un intermezzo comico in italiano, La Pelarina (I729), un precedente di maroné citato nel GDLI, in cui il termine è lemmatizzato nel modo seguente:

Maronè, s.m. sm. Dial. Ciuffo di capelli raccolti e legati con un nastro.

Goldoni, X-7: Politi nel vestire: / abito corto con larghi faldoni, / attillati calzoni alla spagnola, / manica a mezzo braccio, / di bianchi pizzi e fini il manicino, / piccolo cappellino, anzi visibile; / perrucchin col topè, / di dietro il maronè con borsa o coda.

= Voce ven., dal fr. marron nel significato di 'ciuffo di capelli legati con nastro'.

Considerando l'influsso che Goldoni esercitò sul teatro posteriore, è possibile che il librettista di Rossini abbia ripreso il termine da lui. Ma può anche darsi (ed è forse più probabile) che si trattasse di un francesismo in circolazione in italiano nei secc. XVIII e XIX. Il TLFi conferma tra i significati di marron quello segnalato nel GDLI: 
HIST. DE LA COIFFURE. Grosse boucle de cheveux arrondie et nouée d'un ruban.

M a maroné potrebbe essere, più verosimilmente, l'adattamento di marroné, participio passato del verbo, registrato sempre nel TLFi:

Marronner, verbe trans., coiffure, vx. Coiffer (les cheveux) en grosses boucles.

In definitiva, invitiamo i nostri amici traduttori a considerare muso franco equivalente di 'faccia risoluta' e maroné corrispondente a 'boccolo' (confessando che la ricerca è servita anche a noi...).

\section{Cita come:}

Paolo D'Achille, Muso franco e maronè: dubbi lessicali da un Figaro all'altro, "Italiano digitale", 2019, XI, 2019/4 (ottobre-dicembre)

DOI: $10.35948 / 2532-9006 / 2020.3255$

Copyright 2019 Accademia della Crusca

Pubblicato con licenza creative commons CC BY-NC-ND 